\title{
Small Supernumerary Ring Chromosome Derived from an Inverted Duplication of 13q11.2q14 in a Fetus with Coarctation of the Aorta
}

\author{
Jian Zeng Mingyan Huang Juan Lin Xiao Zhang Fenghua Lan
}

Center for Molecular Diagnosis of Genetic Diseases, Dongfang Hospital, Xiamen University Medical College, Fuzhou, China

\section{Established Facts}

- Ring-shaped small supernumerary marker chromosomes derived from an inverted duplication of chromosome $13 \mathrm{q}$ are rare cytogenetic findings with highly heterogeneous phenotypes.

- Very few patients with a chromosomal trisomy or tetrasomy for the most proximal region 13q11.2 q14 have been described.

\section{Novel Insights}

- This is the first prenatal case with an inverted duplication of the most proximal region 13q11q14.11 in a ring chromosome not previously described.

- The findings we presented here deepen our understanding of the clinical consequences of a small supernumerary ring-shaped chromosome originating from an inverted duplication of the most proximal region 13q11q14.11 and may be of help for further studies of critical regions in chromosome 13.

\section{Keywords}

Inverted duplication · Prenatal diagnosis · Ring chromosome - Supernumerary marker chromosomes .

Tetrasomy13q11.2q14

\section{Abstract}

Here, we report a molecular characterization of a small supernumerary marker chromosome (sSMC) derived from the most proximal region of $13 q$ present in a fetus with coarcta- tion of the aorta at ultrasound examination during prenatal diagnosis. Cultured umbilical cord blood cells showed a de novo extra ring-shaped sSMC in 76\% of the cells using a standard banding technique. SNP array revealed a tetrasomy of about 28.4 $\mathrm{Mb}$ in the long arm of chromosome 13 from band $13 q 11$ to $13 q 14.11$ in the fetus's cells. Metaphase/interphase FISH using specific probes located at 13q11, 13q12.11, and

\section{J.Z and M.H. contributed equally to this work.}

\section{KARGER}

(c) 2019 S. Karger AG, Basel

E-Mail karger@karger.com www.karger.com/cgr
Jian Zeng

Center for Molecular Diagnosis of Genetic Diseases, Dongfang Hospital

Xiamen University Medical College, 156 Xi'erhuanbei Road

Fuzhou, Fujian 350025 (China)

E-Mail zengjian1980222@163.com 
$13 q 14.11$, respectively, demonstrated that the supernumerary ring chromosome was derived from an inverted duplication of the region $13 q 11$ q14.11 with a conventional centromere. To the best of our knowledge, this is the first time that an inverted duplication of the most proximal region $13 q 11 q 14.11$ in a ring chromosome is characterized. The findings we presented here deepen our understanding of the clinical consequences of tetrasomy in this region and may be of help for further studies of critical regions in chromosome 13.

(C) 2019 S. Karger AG, Basel

Small supernumerary marker chromosomes (sSMC) consist of structurally rearranged chromosomes that cannot be characterized by conventional cytogenetic techniques. SSMC was observed in $0.075 \%$ of the prenatal cases and $0.044 \%$ of the newborn cases [Liehr et al., 2004, 2007] with an estimated risk of an abnormal phenotype of $7 \%$ for acrocentric chromosomes and $28 \%$ for nonacrocentric chromosomes [Graf et al., 2006]. sSMC can be derived from various chromosomes. Ring chromosome formation associated with an inverted duplication has only rarely been described for sSMC. For chromosome 13, only a few cases have been reported associated with a ring chromosome so far, and most cases were identified as analphoid SSMC derived from an inverted duplication of the most distal region 13q. Thus, very rare patients with a chromosomal trisomy or tetrasomy for the most proximal region of 13q11q14 have been described. Furthermore, the genotype/phenotype correlation with polysomy for certain fragments of chromosome 13 has not been established yet.

Here, we report a molecular characterization of a ringshaped sSMC derived from an inverted duplication of the most proximal region $13 \mathrm{q} 11 \mathrm{q} 14$ during prenatal diagnosis. We also discuss the association between phenotypic features and overexpression of critical regions or genes in the proximal region of chromosome 13 in our case and the previous patients with partial trisomy or tetrasomy 13q11q14.

\section{Clinical Report}

A 26-year-old woman was referred to our center for prenatal cytogenetic diagnosis at approximately 25 weeks' gestation because of a Down syndrome risk of 1/600 from second-trimester maternal serum screening and a high risk of a trisomy 13 in noninvasive prenatal screening by massively parallel DNA sequencing. Ultrasound examination revealed a single fetus with coarctation of the aorta; small atrial septum aperture; narrow left umbilical ar- tery, similar to single umbilical artery; umbilical cord attachment point abnormality, and intestinal inner diameter slightly widened. The biparietal diameter measured $82.3 \mathrm{~mm}(<1$ st percentile; normal range: $<10$ th percentile), femur length was $62.8 \mathrm{~mm}(<1$ st percentile; normal range: $<10$ th percentile) and the amniotic fluid index was $177 \mathrm{~mm}$ (normal range: $70-250 \mathrm{~mm}$ ) under ultrasound at 32 weeks gestation. The fetus was the first pregnancy of this nonconsanguineous and healthy couple. After genetic testing and counseling, the parents decided to terminate the pregnancy at 34 weeks' gestation. External examination showed a male fetus with normal phenotype, but an autopsy was not performed.

\section{Materials and Methods}

\section{Cytogenetic Analyses}

G-banding was performed from cultured umbilical cord blood cells, and parental karyotyping was done from cultured peripheral lymphocytes using standard techniques.

\section{SNP Array}

SNP array was performed on umbilical cord blood cells of the fetus using Affymetrix CytoScan $750 \mathrm{~K}$ Array (Affymetrix Inc., Santa Clara, CA, USA) according to the manufacturer's recommendations. The original data generated by Affymetrix GeneChip Scanner was converted into CYCHP file by Affymetrix GeneChip Convert Console, and the file was analyzed by Affymetrix's own software CHAS (Chromosome Analysis Suite), accessing a database for storing and querying segment data and annotations either locally (by a single user) or installed on a server/common workstation (multiperson use).

\section{Fluorescence in situ Hybridization}

Metaphase FISH study was performed using a BAC probe (RP11-54E8) on cultured umbilical cord blood cells of the fetus. The probe can specifically hybridize to the region of 13q11 and 21q11.2. In addition, 2 BAC clones located at 13q12.11 (RP1193G1) and 13q14.11 (RP11-1112O18), respectively, were used on metaphase chromosomes or interphase nuclei of the abovementioned cells of the fetus according to standard protocols.

\section{Results}

Cultured umbilical cord blood cells showed a de novo cytogenetic mosaic ring-shaped sSMC with a constriction in 38 of the metaphases studied $(n=50)$ using a standard banding technique (Fig. 1A, B). The parents showed normal karyotypes.

SNP array revealed a tetrasomy of about $28.4 \mathrm{Mb}$ in the long arm of chromosome 13 from band $13 \mathrm{q} 11$ to q14.11 in the fetus (Fig. 1C), demonstrating that the additional material on the sSMC originated from this region. Uniparental disomy was excluded. Thus, the fetal karyotype was interpreted as arr[hg19] 13q11q14.11(19,436,286-43,269,421)×4. 


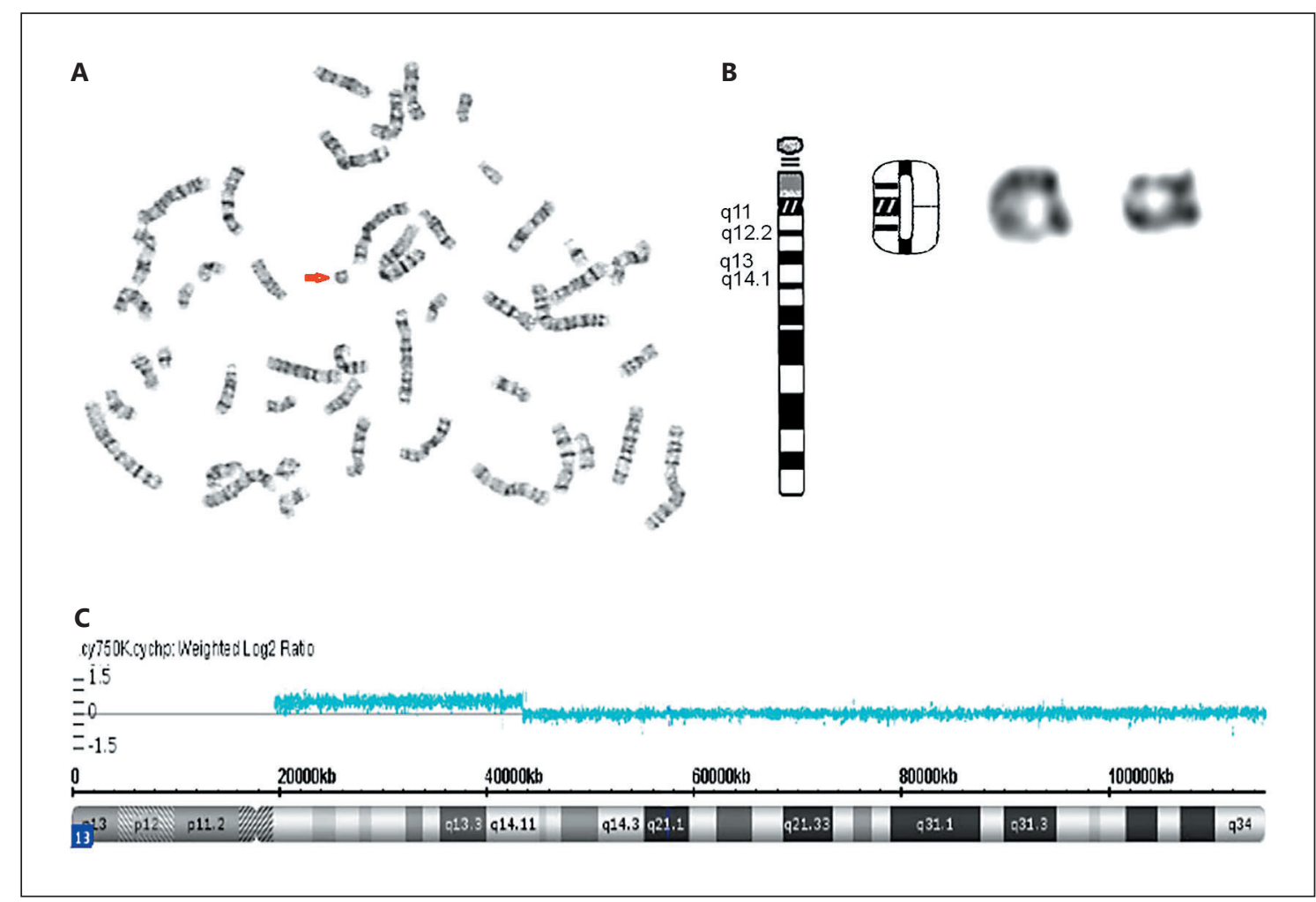

Fig. 1. A Karyotype of the fetus. GTG-banding revealing an sSMC (arrow). B G-banded sSMC of the patient. C SNP-array analysis showing a tetrosomy of about $28.4 \mathrm{Mb}$ in the long arm of chromosome 13 from band $13 \mathrm{q} 11$ to band q14.11 in the fetus.

Using a specific probe (RP11-54E8) for $13 \mathrm{q} 11$ and 21q11.2, metaphase FISH analysis showed 1 positive signal on the ring chromosome (Fig. 2A). For the probe (RP11-93G1) specific to proximal 13q12.11, metaphase and interphase FISH analysis revealed 2 positive green signals on the ring-shaped sSMC in addition to 2 signals on both of the normal chromosomes 13 (Fig. 2B, C), and for the probe (RP11-1112O18) specific to distal $13 q 14.11$, four positive red signals were hybridized in an interphase cell and an enhanced signal was observed on the ring chromosome (Fig. 2D, E). FISH using both BAC RP11-93G1 (green) and RP11-1112O18 (red) demonstrated that signals were hybridized to both ends of sSMC (Fig. $2 \mathrm{~F}$ ), suggesting that the supernumerary ring chromosome was derived from an inverted duplication of the region $13 \mathrm{q} 11.2 \mathrm{q} 14.11$ in the fetus. Therefore, the final karyotpye of the fetus was described as: 47,XY,+mar.ish r(13)inv dup(13)(::q11 $\rightarrow$ q14.11::q14.11 $\rightarrow \mathrm{q} 11::)(\mathrm{RP} 11-54 \mathrm{E} 8+, \mathrm{RP} 11-93 \mathrm{G} 1++, \mathrm{RP} 11-$ $1112 \mathrm{O} 18+\mathrm{enh}) / 46, \mathrm{XY}$.

Small Supernumerary Ring Chromosome from 13q11.2q14 Duplication

\section{Discussion}

In this report, we described a case carrying a mosaic ring-shaped sSMC originating from an inverted duplication of the most proximal region 13q11.2q14.11. For sSMC derived from chromosome 13, only few cases have been reported compared to those from other chromosomes. Most cases were identified as analphoid sSMC derived from supernumerary inverted duplication of the most distal region 13q including 13q21, 13q31, 13q32, and 13q34 [Warburton et al., 2000; Li et al., 2002; Haddad et al., 2012] to 13qter. In the sSMC database (http://ssmctl.com/chromosome-13.html), there are 4 prenatal cases with gains of 13pterq12 segments showing normal children. Therefore, it was considered that the probably nondosage sensitive or uncritical pericentric region of chromosome 13 was located in 13pterq12.11. In our case, the marker was derived from the $13 \mathrm{q} 11 \mathrm{q} 14$ region, indicating that the imbalance of the 13q12.12q14 region might be involved in the fetus's symptoms. 

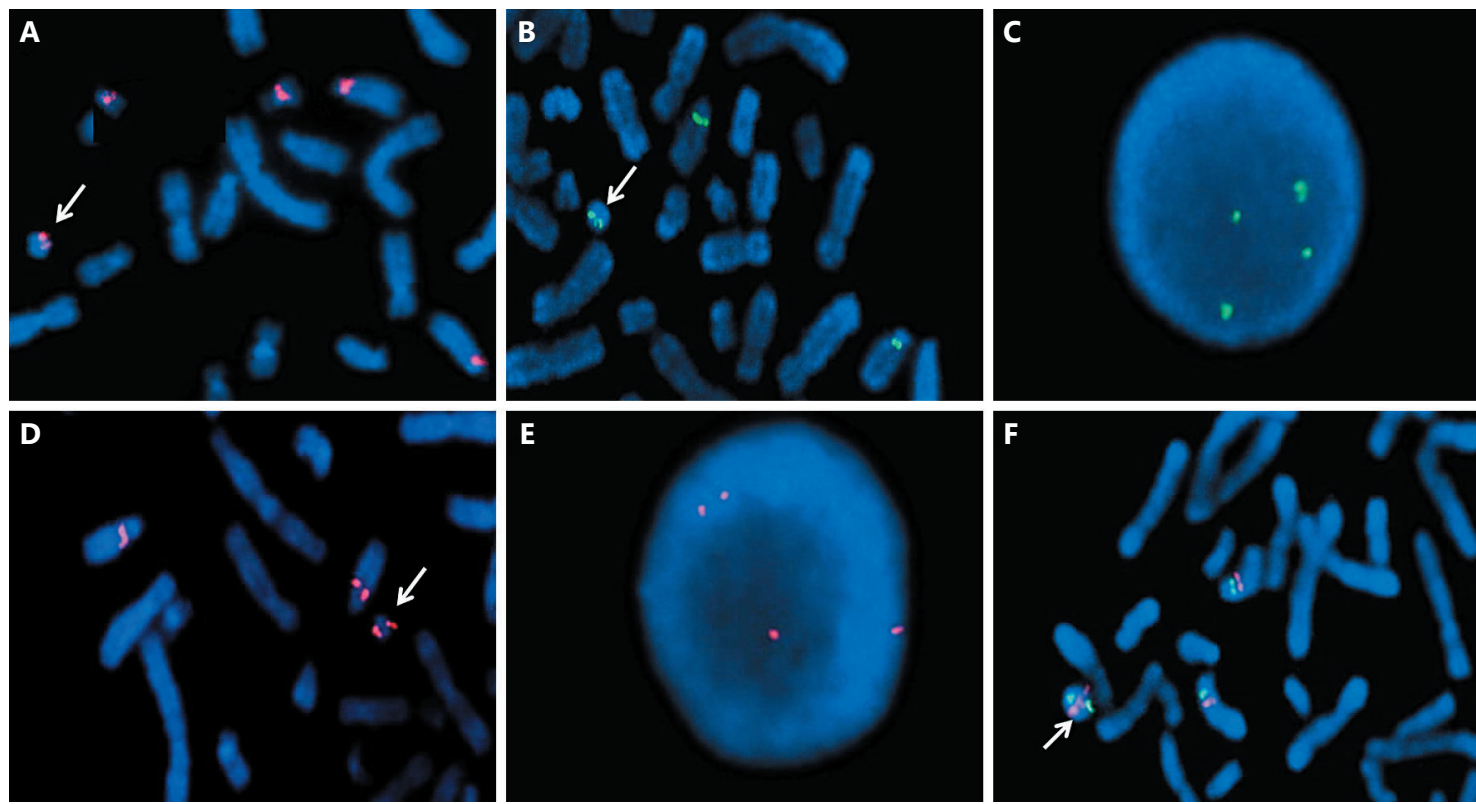

Fig. 2. A-F FISH analysis of the sSMC. Metaphase FISH results using the RP11-54E8 probe (red) showing 1 positive signal on SSMC (A) and the RP11-93G1 probe (green) showing 2 positive signals on sSMC (B). C Interphase FISH results using the RP11-93G1 probe (green) showing 4 positive signals in one cell. D Metaphase FISH results using RP11-1112018 probe (red) showing 1 enhanced

To date, there are 14 cases which are definitely or possibly pathogenic with a gain of a similar or smaller proximal region of 13q11q14 in the DECIPER database (http:// decipher.sanger.ac.uk/). Eight cases in the ISCA database showed developmental delay, intellectual disability, and other clinical features such as autism and ventricular septal defect. According to the literature and databases, 4 cases with pure proximal partial trisomy of the long arm of chromosome 13,13q12q14, with clinical findings have been described (Table 1) [Hermann et al., 2000; Baldwin et al., 2008; Neill et al., 2010; Yu et al., 2012]. In general, the majority of these patients with a proximal trisomy displayed milder dysmorphism and milder intellectual disability as compared to the patients with trisomy or tetrosomy of the distal chromosome segments. Only one patient described by Baldwin et al. [2008] showed multiple anomalies. These severe clinical findings may be due to the more complex chromosome arrangements which were not detected by Gbanding and limited FISH techniques.

Previous studies suggest that the clinical features of tetrasomy of an autosomal segment are similar, but more severe than those of trisomy of the same segment. Furthermore, for some segments $(8 p, 12 p)$, tetrasomy is in positive signal on sSMC. E Interphase FISH results using RP111112018 probe (red) showing 4 positive signals in one cell. $\mathbf{F}$ Metaphase FISH results using both RP11-93G1 (green) and RP111112018 (red) probes showing 2 green signals hybridized to one end of the marker and 2 red green signals hybridized to the other end of the marker. White arrows indicate sSMC. mosaic state, while trisomy without mosaicism is compatible with survival [Brecevic et al., 2000]. The fetus we report here was identified to be a tetrasomy of 13q11q14.11 in mosaic state. A girl with a duplication of the similar region 13q12q14 also displayed coarctation of the aorta as the fetus in our study [Hermann et al., 2000], suggesting that the critical region of 13q12q14 may be involved in the phenotype.

By consulting the OMIM database, there are 28 morbid genes located in the region of 13q11q14.11. Among these genes, B3GLCT (beta-3-glucosyltransferase; OMIM 610308) gene (located at 13q12.3) mutations were found to cause Peters-plus syndrome (OMIM 261540), which is an autosomal recessive disorder characterized by a variety of anterior chamber defects accompanied by some other symptoms including heart defects such as subvalvular aortic stenosis [Maillette de Buy Wenniger-Prick and Hennekam, 2002]. Although the B3GALT gene may not be dosage sensitive, the possibility of mutations in this gene could not be ruled out. Unfortunately, the family refused autopsy. Consequently, we are not able to report other dysmorphic features or internal anomalies not detectable using ultrasound. 
Table 1. Summary of the cases with clinical findings with pure proximal partial long arm duplication/trisomy/tetrasomy of 13q11q14

\begin{tabular}{|c|c|c|c|c|c|}
\hline This study & $\mathrm{M} /$ prenatal & $\begin{array}{l}\text { G-banding, FISH, } \\
\text { SNP-array }\end{array}$ & $\begin{array}{l}47, \mathrm{XY},+\mathrm{mar} \\
(50-70 \%)\end{array}$ & $\begin{array}{l}\mathrm{r}(13) \operatorname{inv} \operatorname{dup}(13) \\
(:: \mathrm{q} 11 \rightarrow \mathrm{q} 14.11:: \mathrm{q} 14.11 \rightarrow \mathrm{q} 11::)\end{array}$ & $\begin{array}{l}\text { Coarctation of the aorta, small atrial } \\
\text { septum aperture, left umbilical artery, } \\
\text { single and narrow left umbilical artery, } \\
\text { umbilical artery }\end{array}$ \\
\hline $\begin{array}{l}\text { Hermann et al., } \\
2000\end{array}$ & $\mathrm{~F} / 7$ & G-banding, FISH & $\begin{array}{l}\text { 46,XX, dup }(13) \\
(100 \%)\end{array}$ & $\operatorname{dup}(13)(\mathrm{q} 12 \rightarrow \mathrm{q} 14)$ & $\begin{array}{l}\text { Short stature, mental retardation, } \\
\text { coarctation of the aorta }\end{array}$ \\
\hline Neill et al., 2010 & F/postnatal & $\begin{array}{l}\text { G-banding, } \\
\text { array CGH }\end{array}$ & $\begin{array}{l}47, \mathrm{XX},+\mathrm{mar} \\
(100 \%)\end{array}$ & $\min (13)($ pter $\rightarrow q 12.12:)$ & Abnormal $^{\mathrm{a}}$ \\
\hline
\end{tabular}

sSMC, small supernumerary marker chromosome. ${ }^{\text {a }}$ No specific description was provided in the study by Neill et al. [2010].

To identify the possible structure of this SMC, we used 3 BAC probes to analyze the fetus's cells. Since an alphasatellite centromere probe specific for chromosome 13 was not commercially available, we used a single-copy $\mathrm{BAC}$ probe (RP11-54E8). It can specifically hybridize to the region of 13q11 and 21q11.2. The region of 13q11 is composed of heterochromatin which is usually repetitive and forms structural functions such as centromeres or telomeres and mainly consists of genetically inactive satellite sequences. In addition, the extra SMC showed an obvious constriction using a standard banding technique. Therefore, we considered the region $13 \mathrm{q} 11$ was composed of constitutive centromere indicating that the ring chromosome included at least part of a conventional centromere.

Inverted duplications with terminal deletion rearrangements have been identified in various chromosomes, but rarely in ring chromosomes. For chromosome 13, only a few cases have been reported to date, less frequently associated with ring chromosomes [Quelin et al., 2014]. To the best of our knowledge, this is the first time an inverted duplication of the most proximal region 13q11.2q14.11 in a ring chromosome is characterized. It has been proposed that at meiosis I, an intrachromosomal U-type recombination or a loop formation combined with 1 or 2 recombination events between homologous alleles had occurred. The resulting recombinant dicentric chromosome may be deleted over the distal recombination site. During meiosis II, a segregation to the opposite poles occurred between the 2 linked chromatids that caused a breakage between the 2 centromeres [Knijnenburg et al., 2007]. Rossi et al. [2008] proposed that a different formation mechanism is based on ring chromosomes: inversion duplication and terminal deletion (inv dup del) of a chromosome circularizes to stabilize itself. The mechanism was proposed to be a multistep process: the formation of the dicentric choromosome at prezygotic event, the inv dup del chromosome formation due to a postzygotic breakage, and the circularization of the inv dup del in some cells. It turned out that these rings are present in most of the cells, but no cells contain either the inv dup del or the simply deleted chromosome, assuming an early selection versus the most viable cell line(s) [Rossi et al., 2008]. When a normal chromosome is taken place by the inv dup del ring chromosome, it is important to consider the effect on clinical implications which is not only due to the simple deletion as generally assumed for ring chromosomes, but also to the associated duplication. But in our case, only the duplication effect on the phenotype should be considered in addition to 46 normal chromosomes.

In conclusion, for the first time, we characterize an inverted duplication of the most proximal region $13 \mathrm{q} 11 \mathrm{q} 14.11$ in a ring chromosome in a fetus with coarctation of the aorta. Although it is difficult to define a clear genotype-phenotype correlation, the findings we presented here deepen our understanding of the clinical consequences of a small supernumerary ring chromosome derived from an inverted duplication of the most proximal region 13q11q14.11 and may be of help for further studies of critical regions in chromosome 13. 


\section{Acknowledgment}

We would like to thank the patient and her family for their participation in this study.

\section{Statement of Ethics}

Written informed consent was obtained from the patient and her husband. The authors have no ethical conflicts to disclose.

\section{Disclosure Statement}

The authors declare no conflicts of interest.

\section{Funding Sources}

This work was supported by the Natural Science Foundation of Fujian Province (grant 2018J01230).

\section{References}

Baldwin EL, May LF, Justice AN, Martin CL, Ledbetter DH: Mechanisms and consequences of small supernumerary marker chromosomes: from Barbara McClintock to modern geneticcounseling issues. Am J Hum Genet 82:398410 (2008).

-Brecevic L, Basaran S, Dutly F, Röthlisberger B, Schinzel A, et al: Tandem triplication of chromosome 13q14 with inverted interstitial segment in a 4-year-old girl. J Med Genet 37: 964-967 (2000).

- Graf MD, Christ L, Mascarello JT, Mowrey P, Pettenati M, et al: Redefining the risks of prenatally ascertained supernumerary marker chromosomes: a collaborative study. J Med Genet 43:660-664 (2006).

-Haddad V, Aboura A, Tosca L, Guediche N, Mas $\mathrm{AE}$, et al: Tetrasomy 13q31.1qter due to an inverted duplicated neocentric marker chromosome in a fetus with multiple malformations. Am J Med Genet A 158A:894-900 (2012).

-Hermann R, Soltész G, Morava E, Kosztolányi G, Czakó M: De novo partial duplication of long arm of chromosome 13: dup(13)(q12 $\rightarrow$ q14). Am J Med Genet 92:296-297 (2000).
Knijnenburg J, van Haeringen A, Hansson KB, Lankester A, Smit MJ, et al: Ring chromosome formation as a novel escape mechanism in patients with inverted duplication and terminal deletion. Eur J Hum Genet 15:548-555 (2007).

S, Malafiej P, Levy B, Mahmood R, Field M, et al: Chromosome 13q neocentromeres: molecular cytogenetic characterization of three additional cases and clinical spectrum. Am J Med Gene 110:258-267 (2002).

Liehr T, Claussen U, Starke H: Small supernumerary marker chromosomes (sSMC) in humans. Cytogenet Genome Res 107:55-67 (2004).

Liehr T, Utine GE, Trautmann U, Rauch A, Kuechler A, et al: Neocentric small supernumerary marker chromosomes (sSMC) - three more cases and review of the literature. Cytogenet Genome Res 118:31-37 (2007).

Maillette de Buy Wenniger-Prick LJ, Hennekam RC: The Peters' plus syndrome: a review. Ann Genet 45:97-103 (2002).

Neill NJ, Torchia BS, Bejjani BA, Shaffer LG, Ballif BC: Comparative analysis of copy number detection by whole-genome BAC and oligonucleotide array CGH. Mol Cytogenet 3:11 (2010).
Quelin C, Spaggiari E, Khung-Savatovsky S, Dupont $\mathrm{C}$, Pasquier L, et al: Inversion duplication deletions involving the long arm of chromosome 13: phenotypic description of additional three fetuses and genotype-phenotype correlation. Am J Med Genet A 164:25042509 (2014).

Rossi E, Riegel M, Messa J, Gimelli S, Maraschio $\mathrm{P}$, et al: Duplications in addition to terminal deletions are present in a proportion of ring chromosomes: clues to the mechanisms of formation. J Med Genet 45:147-154 (2008).

Warburton PE, Dolled M, Mahmood R, Alonso A, Li S, et al: Molecular cytogenetic analysis of eight inversion duplications of human chromosome $13 \mathrm{q}$ that each contain a neocentromere. Am J Hum Genet 66:1794-1806 (2000).

Yu S, Fiedler SD, Brawner SJ, Joyce JM, Zhou XG, Liu HY: Characterizing small supernumerary marker chromosomes with combination of multiple techniques. Cytogenet Genome Res 136:6-14 (2012). 